Isabelle Touwendpoulimdé Kiendrebeogo, Abdou Azaque Zoure», Pegdwendé Abel Sorgho, Albert Théophane Yonli, Florencia Wendkuuni Djigma, Abdoul Karim Ouattara*, Herman Karim Sombie, Sessi Frida Tovo, Edwige T. Yelemkoure, Aboubacar Hierrhum Bambara, Alexis Yobi Sawadogo, Youssef Bakri, Jacques Simpore

\title{
Glutathione S-transferase M1 (GSTM1) and T1 (GSTT1) variants and breast cancer risk in Burkina
}

\section{Faso}

https://doi.org/10.1515/bmc-2019-0020

received July 1, 2019; accepted September 2, 2019.

\begin{abstract}
Background and objective: Breast cancer remains the most common cause of cancer mortality in women. The aim of this study was to investigate associations between genetic variability in GSTM1 and GSTT1 and susceptibility to breast cancer.

Methods: Genomic DNA was extracted from blood samples for 80 cases of histologically diagnosed breast cancer and 100 control subjects. Genotyping analyses were performed by PCR-based methods. Associations between specific genotypes and the development of breast cancer
\end{abstract}

\footnotetext{
*Corresponding author: Abdoul Karim Ouattara, Laboratory of Molecular Biology and Genetics (LABIOGENE), UFR/SVT, University Joseph Ki-Zerbo, 03 P.0. Box 7021, Ouagadougou 03, Burkina Faso; Pietro Annigoni Biomolecular Research Center (CERBA), 01 P.O. Box 364 , Ouagadougou 01, Burkina Faso, E-mail: ak.ouattara02@gmail.com Isabelle Touwendpoulimdé Kiendrebeogo, Pegdwendé Abel Sorgho, Albert Théophane Yonli, Florencia Wendkuuni Djigma, Herman Karim Sombie, Sessi Frida Tovo, Edwige T. Yelemkoure, Laboratory of Molecular Biology and Genetics (LABIOGENE), UFR/SVT, University Joseph Ki-Zerbo, 03 P.0. Box 7021, Ouagadougou 03, Burkina Faso; Pietro Annigoni Biomolecular Research Center (CERBA), 01 P.0. Box 364 , Ouagadougou 01, Burkina Faso

Abdou Azaque Zoure, Laboratory of Molecular Biology and Genetics (LABIOGENE), UFR/SVT, University Joseph Ki-Zerbo, 03 P.O. Box 7021, Ouagadougou 03, Burkina Faso; Institute of Health Sciences Research, (IRSS/CNRST)/ Department of Biomedical and Public Health, 03 BP 7192 Ouagadougou 03, Burkina Faso

Aboubacar Hierrhum Bambara, Service of oncology, University Hospital Yalgado OUEDRAOGO, University Joseph KI ZERBO, UFR/SDS, Ouagadougou, Burkina Faso

Alexis Yobi Sawadogo, Service of Gynecology, University Hospital Yalgado OUEDRAOGO, University Joseph KI ZERBO, UFR/SDS, Ouagadougou, Burkina Faso Youssef Bakri, Laboratoire de Biologie des Pathologies HumainesBioPatH. Faculty of Sciences, University of Mohammed V-Rabat, Morocco $\psi=$ Co-first author
}

were examined using logistic regression to calculate odds ratios [1] and 95\% confidence intervals (95\% CI).

Results: No correlation was found between GSTM1null and breast cancer (OR $=1.83$; 95\%CI 0.90-3.71; $\mathrm{p}=$ 0.10), while GSTT1-null (OR $=2.42$; 95\%CI 1.17-5.02; $\mathrm{p}=$ 0.01 ) was associated with increased breast cancer risk. The GSTM1/GSTT1 double null was not associated with an increased risk of developing breast cancer $(\mathrm{OR}=2.52$; 95\%CI 0.75-8.45; $p=0.20$ ). Furthermore, analysis found no association between GSTM1-null (OR $=1.12$; 95\%CI 0.08$15.50 ; p=1.00$ ) or GSTT1-null (OR = 1.71; 95\%CI 0.13-22.51; $p=1.00$ ) and the disease stage of familial breast cancer patients or sporadic breast cancer patients (GSTM1 (OR $=0.40 ; 95 \%$ CI 0.12-1.32; $p=0.20)$ and GSTT1 (OR = 1.41; 95\%CI 0.39-5.12; $p=0.75)$ ). Also, body mass index (BMI) was not associated with increased or decreased breast cancer risk in either GSTM1-null (OR $=0.60$; 95\%CI 0.21$1.68 ; p=0.44)$ or GSTT1-null (OR $=0.60$; 95\%CI 0.21-1.68; $p=0.45)$.

Conclusion: Our results suggest that only GSTT1-null is associated with increased susceptibility to breast cancer development.

Keywords: GSTM1-GSTT1, Genotypes, Breast cancer risk, Burkina Faso

\section{Introduction}

According to GLOBOCAN 2018 (Cancer database), there are about 2.1 million newly diagnosed female breast cancer cases worldwide each year, making up $11.6 \%$ of all cancer diagnoses. In 2018, breast cancer was the cause of 626,679 (6.6\%) deaths, accounting for almost 1 in 4 cancer cases among women [2]. Thus, breast cancer is the most common 
cause of cancer mortality in women, and it constitutes an important health problem [2]. Research has suggested that risk factors for breast cancer are environmental, genetic, nutritional (diet), and hormonal. Several well-known risk factors for breast cancer development are family history of breast cancer, advanced age, early puberty, late menopause, nulliparity, obesity and hereditary transmission of certain predisposition genes, including the BRCA1 and BRCA2 genes (involved in $5-10 \%$ of breast cancer cases) and the CHEK2, TP53, ATM and PTEN genes $[1,3,4]$. In Burkina Faso, previous studies have described the distribution of breast cancer risk factors in a population [5] and shown that multiparity is associated with a decreased risk of breast cancer [6]. Genetic analyses targeting relevant exons in BRCA1 genes have also been performed [7]. Furthermore, environmental factors have been well-reported in many breast cancers studies. These environmental factors include carcinogens, xenoestrogens and chemical mutagens [3]. In chemical carcinogenesis, three enzyme systems - namely cytochrome P450 (CYP), antioxidant enzymes (AOEs), and glutathione S-transferases (GSTs) -- play an important role [8]. GSTs play a key role in the detoxification of electrophiles and potentially carcinogenic compounds by glutathione conjugation $[9,10]$. In mammalian tissues, seven common classes of cytosolic GST enzymes have been identified (GST classes alpha $(\alpha), \operatorname{mu}(\mu), \operatorname{pi}(\pi)$, omega $(\omega)$, theta $(\theta)$, $\operatorname{sigma}(\sigma)$ and zeta $(\zeta))$, and each class is encoded by a separate gene or gene family (respectively, GSTA, GSTM, GSTP, GSTO, GSTS, GSTT and GSTZ genes) [9, 11-13].

Many GST genes are polymorphic; thus, particular allelic variants are associated with altered risk (or outcome) of a variety of diseases [10]. These polymorphic variants in GST genes have been reported in different populations [4].

It has been demonstrated that GSTP protein level and GST activity in tumor tissue are significantly higher than in normal breast tissue $[14,15]$. GSTs are also cancer chemotherapeutic agents, and thus contribute to tumor resistance to these agents [16]. Therefore, GSTs may be of clinical value in the case of some malignant cancers [17]. The $\mu$ (GSTM1:chromosome1p13.3) and $\theta$ (GSTT1: chromosome 22q11.23) members of this multigene family are candidate cancer susceptibility genes because of their ability to regulate the conjugation of carcinogenic compounds to excretable hydrophilic metabolites [8, 18]. GSTM1-null or GSTT1-null might then increase risk for deleterious effects of exposure to a wide range of environmental carcinogens [19]. Both variants are homozygous deletions (null genotype) and are therefore associated with the loss of enzyme activity and increased vulnerability to cytogenetic damage [16]. Previous findings have provided evidence that variants with GSTP1 and GSTM1 polymorphisms could influence breast cancer risk, response to chemotherapy, and overall survival in breast cancer patients treated with chemotherapy [20].

Rebbeck et al., (1997) have demonstrated that GSTM1 and GSTT1 are associated with variability in age at first breast cancer diagnosis in BRCA1 mutation carriers, with $22 \%$ difference across the observed age range (25-40 years) explained by the GSTT1 genotype [4]. Studies on polymorphisms in GSTM1 and GSTT1 showed a prevalence of $27.8 \%$ of GSTM1-null and $46.8 \%$ of GSTT1-null in Cameroun , 48.8\% of GSTM1-null and 37.3\% of GSTT1-null in Ethiopia , 49.2\% of GSTM1-null and 28.3\% of GSTT1null in Italy, and 55.3\% of GSTM1-null and 27.7\% of GSTT1null in Spain [21]. Other research has found that GSTM1null individuals may have an increased risk of recurrent pregnancy loss [22], and an Indian meta-analysis found that female carriers of GSTT1 and GSTM1-null genotypes have a higher frequency of pregnancy loss [23].

Based on studies investigating the association of GSTT1 and GSTM1 variants with breast cancer risk, as well as on the role of GSTs in inactivating endogenous metabolites during oxidative stress and its influence on the normal functions of mammalian tissues, we investigated the distribution of GSTM1 and GSTT1 variants in patients with histologically diagnosed breast cancer in comparison to controls to explore the possible association of GST genotypes and risk of breast cancer development. The present case-control study is based on data acquired from a population in Burkina Faso.

\section{Materials and methods}

\section{Study population and sample collection}

This cross-sectional study was conducted from October 2017 to June 2018 in Burkina Faso. We enrolled 80 subjects with histologically diagnosed breast cancer (Services of Oncology and Gynecology, University Hospital Center (CHU-Yalgado OUEDRAOGO) and 100 healthy subjects without breast cancer (Service Gynecology). All female patients with breast tumors confirmed by anatomopathological test were included as cases, and all female subjects without any breast anomaly (as confirmed by mammography) were included as controls. Familial cases were defined as patients with first or second-degree 


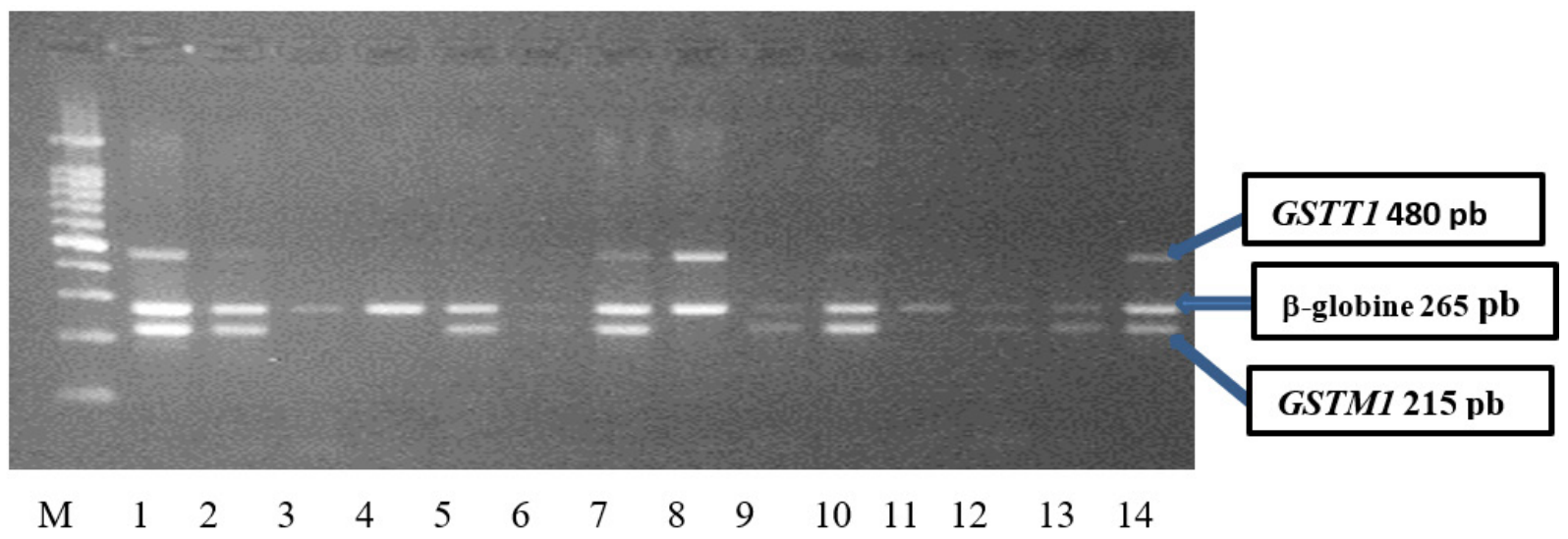

Figure 1: PCR-multiplex electrophoresis gel. $\mathbf{M}=$ Molecular weight marker (100 bp); 1; 7 et 14 = Double present genotypes of GSTM1 and GSTT1; 2; 5; 10; 12 et 13 = Genotypes of GSTM1 present and GSTT1 null; 3; 4; 11= Double genotypes null of GSTM1 and GSTT1; 6; = invalid; 8 GSTM1 null and GSTT1 present.

relatives in the same familial branch who had been diagnosed with breast cancer at any age.

All patients included in the study freely consented to their participation Bedridden patients and control cases with family cancer history were excluded from the study. Fasting state was not part of our inclusion or exclusion criteria. Sampling was performed using two types of tubes, a $5 \mathrm{ml}$ violet EDTA tube and a $5 \mathrm{ml}$ red dry tube. After patients provided informed consent, approximately $10 \mathrm{~mL}$ of venous blood was collected into both the EDTA tube and the dry tube. Whole blood was used immediately for genomic DNA extraction. The samples were then centrifuged at $3500 \mathrm{~g}$ for 15 minutes to collect the plasma and serum, which were then stored at $-20^{\circ} \mathrm{C}$ at the Pietro Annigoni Biomolecular Research Center (CERBA) for further processing.

Breast cancer patients were noted as having a "family history of breast cancer" if at least one member of their family (niece, sister, mother, cousin, etc.) had or currently has breast cancer. Breast cancer patients were considered to have a "family history of other cancer type" if at least one member of their family had or currently has any cancer other than breast cancer. Breast cancer patients were classified as "sporadic breast cancer cases" if no one in their family had or currently has breast cancer.

\section{Collection of epidemiological and clinical data}

Sociodemographic data including clinical examination and medical follow-up of patients was collected on an individual sheet.

\section{Extraction of genomic DNA and Characterization of deletions of GSTM1 and GSTT1 genes by multiplex PCR.}

Genomic DNA was extracted from whole blood using the "DNA Rapid Salting-Out" method described by [24] and stored at $-80^{\circ} \mathrm{C}$ until use. Multiplex PCR was performed on the GeneAmp PCR system 9700 (Applied Biosystem, USA) according to the method described by [25] with a reaction volume of $25 \mu \mathrm{L}$, including $10 \mu \mathrm{L}$ of Maxter Mix Ampli Taq Gold $\AA$ (Applied Biosystems, USA), $1 \mu \mathrm{L}$ of each primer pair $(20 \mu \mathrm{M})$ (Applied Biosystems, USA) GSTM1 ( Forward5'-GAACTCCCTGAAAAGCTAAAGC-3'; Reverse 5'GTTGGGCTCAAATATACGGTGG-3') GSTT1 (Forward 5' TTCCTTACTGGTCCTCACATCTC-3', Reverse 5'-TCACCGGATCATGGCCAGCA-3'), 7 $\mu \mathrm{L}$ of nuclease-free water, and $2 \mu \mathrm{L}$ of DNA. All reagents were purchased from Applied Biosystem (ABI, Appleura International Inc., Foster City, CA, USA). The amplification program was as follows: an activation phase at $94^{\circ} \mathrm{C}$ for $5 \mathrm{~min} ; 40$ cycles of a series of denaturation at $94^{\circ} \mathrm{C}$ for $1 \mathrm{~min}$, hybridization at $57^{\circ} \mathrm{C}$ for $1 \mathrm{~min}$, elongation at $72^{\circ} \mathrm{C}$ for $1 \mathrm{~min}$; and extension at $72^{\circ} \mathrm{C}$ for $7 \mathrm{~min}$. The PCR products were subjected to $2 \%$ agarose gel electrophoresis and visualized under UV light at $312 \mathrm{~nm}$ using the Gene Flash rRevelation (Syngenge Bio Imaging, USA) PCR amplification was considered successful if the sample had a band corresponding to b-globin gene fragment (5'-CAACTTCATCCACGTTCACC-3', 5'-GAAGAGCCAAGGACAGGTAC-3') (Figure 1).

Ethical approval: The research related to human use has been complied with all the relevant national regulations, institutional policies and in accordance the 
Table 1: General Characteristics of the case group.

\begin{tabular}{llll}
\hline Variable & $\mathbf{N}=\mathbf{8 0}(\%)$ & Variable & $\mathbf{N}^{2}=\mathbf{8 0}(\%)$ \\
\hline Age & & Body mass index(BMI) & \\
Mean & $48.20 \pm 12.40$ years & Normal/lean $<25 \mathrm{~kg} / \mathrm{m}^{2}$ & $49(61.25 \%)$ \\
Range & $28-80$ years & Overweight between 25 and $30 \mathrm{~kg} / \mathrm{m}^{2}$ & $21(26.25 \%)$ \\
& & Obese $\geq 30 \mathrm{~kg} / \mathrm{m}^{2}$ & $10(12.50 \%)$ \\
Histological type & & Stage & \\
Ductal IDC & $75(93.75 \%)$ & I & $5(6.25 \%)$ \\
Lobular ILC & $1(1.25 \%)$ & IIA/IIB & $35(43.75 \%)$ \\
Others & $4(5 \%)$ & IIIA/IIIB & $23(28.75 \%)$ \\
& & IV & $17(21.25 \%)$ \\
Family history of & & Family history of other cancer type & \\
breast cancer & $15(18.75 \%)$ & & $13(16.25 \%)$ \\
Yes & $65(81.25 \%)$ & Yes & $67(83.75 \%)$ \\
No & & No & \\
\hline
\end{tabular}

IDC: Invasive Ductal Carcinoma; ILC: Invasive Lobular Carcinoma

tenets of the Helsinki Declaration, and has been approved by the Burkina Faso Health Research Ethics Committee.

Informed consent: Informed consent has been obtained from all individuals included in this study

\section{Statistical analysis}

Data were analyzed using the standard Statistical Package for Social Sciences (SPSS) software version 20.0 for Windows and EPI Info software version 7.1.

The $\chi 2$ test was used to calculate the difference in the genotype distributions. Relative risk was estimated with Odds Ratio (OR) and the Cornfield 95\% confidence interval (95\%CI). P values below 0.05 or Odds Ratios with a 95\%CI were considered statistically significant.

The quantitative variables were expressed as mean \pm standard deviation, and comparisons between groups were made with the Student's t-test.

Associations between allelic variants and cancer were established by comparing frequencies between cases and controls using the $\chi 2$ test.

\section{Results}

In this study, we examined the influence of two GST genes, GSTM1 and GSTT1, in breast cancer susceptibility. The study is cross-sectional, including breast cancer patients and healthy females without breast cancer as controls.

\section{General data on study subjects}

The control group had an average age of $25.12 \pm 8.30$ years with a range of 16-37 years, and 100\% were female and urban. In contrast, the mean age of cases was 48.20 \pm 12.40 years (range: $28-80$ years). The cases include $87.50 \%$ urban and $12.50 \%$ rural. According to the criteria of the US National Institute of Health/National Heart Lung and Blood Institute (NCI/NHLBI), 38.75\% of cases were classified as obese or overweight (Table 1). Clinical characteristics show that most histological cases were invasive ductal carcinoma (93.75\%), followed by others $(1.25 \%)$ and invasive lobular carcinoma (1.25\%). Half $(50 \%)$ were in an advanced stage (Stage III or IV).

We used TNM Classification (UICC, 7e Edition, 2009) to describe the cancer types (Sobin, Gospodarowicz et Wittekind, 2009) (Table 1). A proportion of $18.75 \%$ and $16.25 \%$ of the study cases reported a family history of 
Table 2: ORs for GSTM1 and GSTT1 breast cancer.

\begin{tabular}{lllll}
\hline Genotype & $\begin{array}{l}\text { Control } \\
\mathbf{N = 1 0 0}(\%)\end{array}$ & $\begin{array}{l}\text { Breast cancer } \\
\mathbf{N}=\mathbf{8 0}(\%)\end{array}$ & OR $(\mathbf{9 5} \% \mathrm{Cl})$ & P value \\
\hline GSTM1 & & & & \\
Present & $82(82.00 \%)$ & $57(71.25 \%)$ & 1 & 0.10 \\
Null & $18(18.00 \%)$ & $23(28.75 \%)$ & $1.83(0.90-3.71)$ & \\
GSTT1 & & & & \\
Present & $85(85.00 \%)$ & $56(70.00 \%)$ & 1 & $0.01^{*}$ \\
Null & $15(15.00 \%)$ & $24(30.00 \%)$ & $2.42(1.17-5.02)$ & \\
GSTM1/GSTT1 & & & & \\
Double Present & $72(93.51 \%)$ & $40(85.11 \%)$ & 1 & $2.52(0.75-8.45)$ \\
Double Null & $5(6.49 \%)$ & $7(14.89 \%)$ & 20
\end{tabular}

$\mathrm{p}$-value<0.05. ORs (odds ratio); $\mathrm{Cl}$ (confidence interval) from conditional logistic regression.

breast cancer and other cancers (liver, stomach, anus, lung, esophagus, uterus, ovary), respectively (Table1).

\section{Breast cancer risk and GSTM1 or GSTT1}

Genotype distributions of GSTM1 and GSTT1 in cases and controls are summarized in Table 2. GSTM1-null was found in $28.75 \%$ and $18.00 \%$ of the cases and the controls, respectively.

The GSTM1-null genotype was not significantly more common among breast cancer cases as compared to controls ( $\mathrm{OR}=1.83 ; 95 \% \mathrm{CI}$ 0.90-3.71; $\mathrm{p}=0.10)$. In contrast, the GSTT1-null genotype was significantly more frequent in cases $(30.00 \%)$ than in controls $(15.00 \%)(\mathrm{OR}=2.42 ; 95 \% \mathrm{CI}$ 1.17-5.02; $\mathrm{p}=0.01$ ). Lastly, we did not find any significant increase in breast cancer risk associated with GSTM1/ GSTT1 double null genotypes (OR= 2.52; 95\%CI 0.75-8.45; $\mathrm{p}$ $=0.20$ ), with $14.89 \%$ of cases and $6.49 \%$ of controls having GSTM1/GSTT1 double null genotypes.

\section{Disease stage and GSTM1/GSTT1}

The association between presumed risk factors and the disease stage of familial/sporadic breast cancer cases is shown in Table 3. Data was analyzed using logistic regression analysis, which found no association between GSTM1-null or GSTT1-null genotypes and the disease stage of familial breast cancer patients (GSTM1-null OR $=1.12$; 95\%CI 0.08-15.50; $p=1.00$ and GSTT1-null OR =
1.71; $95 \%$ CI $0.13-22.5 ; p=1.00$ ) or sporadic breast cancer patients (GSTM1-null OR $=0.40 ; 95 \%$ CI 0.12-1.32; $p=0.20$ and GSTT1-null OR $=1.41 ; 95 \%$ CI 0.39-5.12; $p=0.75$ ) (Table 3). Likewise, the GSTM1/GSTT1double null genotype was not associated with any differences in disease stage; thus, all genotypes lacked any association with the stage of disease in either familial (OR=0.5; 95\%CI 0.02-11.08; $p$ $=1.00)$ or sporadic breast cancer patients $(\mathrm{OR}=0.29 ; 95 \% \mathrm{CI}$ 0.04-1.77; $p=0.31$.

\section{Body mass index (BMI) and GSTM1/GSTT1}

BMI was not associated with either significantly increased or significantly decreased breast cancer risk. Table 4 shows the risk of breast cancer in combination with GSTM1-null and GSTT1-null genotypes. The respective frequencies in overweight/obese (BMI between 25 and $30 \mathrm{~kg} / \mathrm{m}^{2} ; \mathrm{BMI} \geq 30 \mathrm{~kg} / \mathrm{m}^{2}$ ) and normal/lean (BMI $<25 \mathrm{~kg} /$ $\mathrm{m}^{2}$ ) were $57.89 \%$ and $69.57 \%$ for GSTM1-null (OR=0.60; 95\%CI 0.21-1.68; $p=0.44)$, $45.83 \%$ and $54.17 \%$ for GSTT1null (OR=0.60; 95\%CI 0.21-1.68; $p=0.45)$, and $42.86 \%$ and $57.14 \%$ for GSTM1/GSTT1 double null (OR=1.80; 95\%CI 0.359.14; $p=0.63)$.

\section{Discussion}

The relationship between GSTM1- and GSTT1-null genotypes and breast cancer risk was examined in a population-based case-control study in Burkina Faso. 
Table 3: Association of GSTM1 and GSTT1 genotypes with the disease stage of familial and sporadic breast cancer patients.

\begin{tabular}{|c|c|c|c|c|c|c|c|c|}
\hline \multirow[t]{2}{*}{ Genotype } & \multicolumn{4}{|c|}{ Familial breast cancer cases } & \multicolumn{4}{|c|}{ Sporadic breast cancer cases } \\
\hline & Stage I\&II & Stage III\&IV & OR $(95 \% \mathrm{Cl})$ & Pvalue & Stage I\& II & Stage III\& IV & OR $(95 \% \mathrm{Cl})$ & $P$ value \\
\hline \multicolumn{9}{|l|}{ GSTM1 } \\
\hline Present & $3(27.27 \%)$ & $8(72.73 \%)$ & 1 & \multirow[b]{2}{*}{1.00} & $8(17.78 \%)$ & $37(82.22 \%)$ & 1 & \multirow[b]{2}{*}{0.20} \\
\hline Null & $1(25.00 \%)$ & $3(75.00 \%)$ & $\begin{array}{l}1.12(0.08- \\
15.50)\end{array}$ & & $7(35.00 \%)$ & $13(65.00 \%)$ & $\begin{array}{l}0.40(0.12- \\
1.32)\end{array}$ & \\
\hline \multicolumn{9}{|l|}{ GSTT1 } \\
\hline Present & $3(30 \%)$ & $7(70 \%)$ & 1 & \multirow[b]{2}{*}{1.00} & $11(25.00 \%)$ & $33(75.00 \%)$ & 1 & \multirow[b]{2}{*}{0.75} \\
\hline Null & $1(20.00 \%)$ & $4(80.00 \%)$ & $\begin{array}{l}1.71(0.13- \\
22.51)\end{array}$ & & $4(19.05 \%)$ & 17(80.95\%) & $\begin{array}{l}1.41(0.39- \\
5.12)\end{array}$ & \\
\hline \multicolumn{9}{|c|}{ GSTM1/ GSTT1 } \\
\hline Double & $3(33.33 \%)$ & $6(66.67 \%)$ & 1 & & $7(28.58 \%)$ & $24(77.42 \%)$ & 1 & \multirow{3}{*}{0.31} \\
\hline Present & & & & 1.00 & & & & \\
\hline Double Null & $1(50.00 \%)$ & $1(50.00 \%)$ & $\begin{array}{l}0.5(0.02- \\
11.08)\end{array}$ & & $3(50.00 \%)$ & $3(50.00 \%)$ & $\begin{array}{l}0.29(0.04- \\
1.77) \\
\end{array}$ & \\
\hline
\end{tabular}

The results for our study showed a frequency of $14.89 \%$ of GSTM1/GSTT1 double null, while GSTM1-null occurred in $28.75 \%$ and $18.00 \%$ of the cases and the controls respectively. The GSTT1-nullgenotype was more frequent in cases $(30.00 \%)$ than in controls (15.00\%). Finally, $14.89 \%$ of cases and $6.49 \%$ of controls had a GSTM1/GSTT1 double null genotype. Studies by Garte et al.; (2001) and Bu et al. (2004) found the frequency of the GSTM1-null genotype to be $42-60 \%$ among Caucasians, $42-54 \%$ among Asians, $16-36 \%$ among Africans, and 54.6\% among Arabs. They also found that the frequency of the GSTT1-null genotype was $13-26 \%$ for Caucasians, 35-52\% for Asians, $15-26 \%$ for Africans, and 25\% for Arabs. The frequency of the GSTM1/ GSTT1 double null genotype was $10.4 \%$ in Caucasians, $24.6 \%$ in Asians, and $12.6 \%$ in Africans) [13, 26]. Indeed, our proportions of the GSTM1-null, GSTT1-null and GSTM1/ GSTT1 double null genotypes are relatively close to the reported prevalence in African populations. Furthermore, these two GST variants have been investigated for their association with susceptibility to human cancers such as breast cancer. Often, such studies have resulted in low penetrance or high prevalence associations between cancer risk and the GSTM1/GSTT1 double null variant [9]. In our study, GSTM1 and GSTT1-null genotypes were not associated with increased breast cancer risk. Indeed, on the strong association between GSTM1- and GSTT1-null genetic variants and breast cancer, study results have not yet converged [27-29]. Thus, in a meta-analysis of 10,067 cancer cases and 12,276 controls, GSTM1-null and
GSTT1-null variants are associated with an increased risk of breast cancer in Asians [30]. Also, the GSTM1-null genotype was associated with increased breast cancer risk, but no associations between the GSTT1-null genotype and neoplasia risk were found in populations in Mexico [28]. Likewise, in a Portuguese population, researchers found an increased breast cancer risk associated with GSTM1-null and GSTT1-null genotypes both alone and in combination with GSTP1 valine alleles (rs1695/rs1138272) [31].

Another study has shown an increased breast cancer risk associated with the GSTM1-null genotype, while no association was found between the GSTT1-null genotype and overall breast cancer risk. These results suggest that variants in low penetrance genes ,such as GSTM1, GSTT1 and GSTP1, are associated with an increased breast cancer risk [32]. Indeed in a Turkish study, neither the GSTT1null nor GSTM1-null genotype were associated with a significantly increased risk of developing breast cancer, but a combined genetic variability in members of the GST gene family may be associated with an increased susceptibility to breast cancer [33]. A meta-analysis from China provides strong support for earlier studies, showing no overall association for the GSTM1 and GSTT1 deletion variants [34]. In contrast, in an Indian population, the GSTT1/GSTM1 double null genotype was found to be protective against the development of carcinoma breast and did not show any association with response to chemotherapy. However, tumors more than $5 \mathrm{~cm}$ in size 
Table 4: Association of GSTM1 and GSTT1 genotypes with the body mass index (BMI) in breast cancer patients.

\begin{tabular}{lllll}
\hline Genotype & Normal/lean & Overweight/Obese & OR $(95 \% \mathrm{Cl})$ & $p$ - value \\
\hline GSTM1 & & & & \\
Present & $33(42.11 \%)$ & $24(57.89 \%)$ & 1 & 0.44 \\
Null & $16(69.57 \%)$ & $7(30.43 \%)$ & $0.60(0.21-1.68)$ & \\
GSTT1 & & & & \\
Present & $36(64.29 \%)$ & $20(35.71 \%)$ & 1 & 0.45 \\
Null & $13(54.17 \%)$ & $11(45.83 \%)$ & $1.52(0.57-4.02)$ & \\
GSTM1/GSTT1 & & & & 0.63 \\
Double Present & $23(57.50 \%)$ & $17(42.50 \%)$ & 1 & \\
Double Null & $3(57.14 \%)$ & $4(42.86 \%)$ & $1.80(0.35-9.14)$ & \\
\hline
\end{tabular}

were associated with increased GSTM1 gene expression [35]. Thus our study, which does not find an association between breast cancer risk and these variants, is consistent with some of the results of previous studies.

No association was found between GSTM1- (OR $=1.12$; 95\%CI 0.08-15.50) or GSTT1- (OR $=2.28$; 95\%CI 0.1828.18) null genotypes and the disease stage of familial breast cancer patients or sporadic breast cancer patients (GSTM1, OR $=0.40$; 95\%CI 0.12-1.32) and (GSTT1, OR = 1.41; 95\%CI 0.39-5.12). High penetrance genes and certain low penetrance genes may also play a role in the familial inheritance of breast cancer [29]. In previously conducted studies, no significant associations between GSTM1 or GSTT1 variants and disease stage were observed [19]. GSTM1 deletion was found to be significantly associated only with familial breast cancer; GSTT1 was associated only with sporadic breast cancer. However, familial breast cancer patients with a GSTM1-null genotype had a relatively higher risk of advanced disease stage [29].

In a previous study, the association with breast cancer were also null for GSTM1-null and GSTT1-null in pre- and postmenopausal women, or for early versus advanced stage breast cancer [34]. In the Carolina Breast CancerStudy for women with a history of breast cancer in one or more first-degree relatives, odds ratios were 2.1 ( $95 \%$ confidence interval, 1.0-4.2) for GSTM1-null and 1.9 (0.8-4.6) for GSTT1null genotypes. Among these women with family histories, age at diagnosis was significantly earlier for those with the GSTM1-null genotype [19]. Furthermore, it has been noted that none of the allelic variants associated with sporadic breast cancer were associated with familial breast cancer [29]. Finally, there were no associations found between GSTM1-null (OR=0.60; 95\%CI 0.21-1.68) and GSTT1-null
(OR=0.60; 95\% CI 0.21-1.68) genotypes in overweight/ obese or normal/lean individuals with susceptibility to breast cancer. Few studies have directly addressed the relationship between GSTM1-null and GSTT1-null variants with breast cancer. Nevertheless, previous research has observed relationships between GSTM1 and BMI.

Postmenopausal women null for GSTM1 and having a BMI above $24.47 \mathrm{~kg} / \mathrm{m}^{2}$ have a sevenfold increase in breast cancer risk, while women null for GSTM1 and having a BMI less than $24.47 \mathrm{~kg} / \mathrm{m}^{2}$ were not at increased risk of breast cancer [36]. Our results contradict this data, but corroborate the results of Vogl et al., (2004) who found no association between those GST allelic variants, BMI, and the occurrence of breast cancer [19].

\section{Conclusion}

Our results suggest that no strong association exists between GSTM1-null, GSTT1-null, or GSTM1/GSTT1 double null genotypes and susceptibility to breast cancer development. The association studies between breast cancer risk and GSTM1 or GSTT1 variants could be investigated further, in agreement with most previous studies. The absence of positive associations for GSTM1null and GSTT-null genotypes in women with either a family history of breast cancer or sporadic breast cancer and BMI indicate that further investigation is required to confirm a potential role for GST genotypes in both breast cancer prognosis and response to treatment.

Author's contributions: ITK, AAZ, PAS and ATY designed this study. AAZ, AHB and AYS recruited patients. PAS, 
ATY, ITK, FWD, AKO, HKS, TF and ETY recruited controls cases. ITK, PAS and AAZ carried out the manipulations, statistical analyses and wrote the manuscript. YB and JS revised the manuscript. All authors have read and corrected the manuscript.

Conflict of interest: Authors state no conflict of interest

\section{References}

1. Couch FJ, Wang X, McGuffog L, Lee A, Olswold C, Kuchenbaecker KB, et al. Genome-wide association study in BRCA1 mutation carriers identifies novel loci associated with breast and ovarian cancer risk. PLoS Genet. 2013;9:e1003212.

2. Bray F, Ferlay J, Soerjomataram I, Siegel RL, Torre LA, Jemal A. Global cancer statistics 2018: GLOBOCAN estimates of incidence and mortality worldwide for 36 cancers in 185 countries. CA Cancer J Clin. 2018;68:394-424.

3. Mitrunen K, Hirvonen A. Molecular epidemiology of sporadic breast cancer. The role of polymorphic genes involved in oestrogen biosynthesis and metabolism. Mutat Res. 2003;544:9-41.

4. Martin AM, Weber BL. Genetic and hormonal risk factors in breast cancer. J Natl Cancer Inst. 2000;92:1126-35.

5. Bambara HA, Zoure AA, Sawadogo AY, Ouattara AK, Ouedraogo NLM, Traore SS, et al. Breast cancer: descriptive profile of 80 women attending breast cancer care in the Department of General and Digestive Surgery of CHU-YO. Pan Afr Med J. 2017;28:314.

6. Zoure AA, Bambara AH, Sawadogo AY, Ouattara AK, Ouedraogo M, Traore SS, et al. Multiparity and Breast Cancer Risk Factor among Women in Burkina Faso. Asian Pac J Cancer Prev. 2016;17:5095-5099.

7. Zoure AA, Slaoui M, Bambara HA, Sawadogo AY, Compaore TR, Ouedraogo NLM, et al. BRCA1 c.68_69delAG (exon2), c.181T>G (exon5), c.798_799delTT and 943ins10 (exon11) mutations in Burkina Faso. J Public Health Afr. 2018;9:663.

8. Rebbeck TR. Molecular epidemiology of the human glutathione S-transferase genotypes GSTM1 and GSTT1 in cancer susceptibility. Cancer Epidemiol Biomarkers Prev. 1997;6:73343.

9. Coles BF, Kadlubar FF. Detoxification of electrophilic compounds by glutathione $\mathrm{S}$-transferase catalysis: determinants of individual response to chemical carcinogens and chemotherapeutic drugs? Biofactors. 2003;17:115-30.

10. Strange RC, Spiteri MA, Ramachandran S, Fryer AA. Glutathione-S-transferase family of enzymes. Mutat Res. 2001;482:21-6.

11. Hussey AJ, Hayes JD. Characterization of a human class-Theta glutathione S-transferase with activity towards 1-menaphthyl sulphate. Biochem J. 1992;286 ( Pt 3):929-35.

12. Aliya S, Reddanna P, Thyagaraju K. Does glutathione S-transferase Pi (GST-Pi) a marker protein for cancer? Molecular and Cellular Biochemistry. 2003;253:319-327.
13. Gara S, Abessi M, Bendjemena K, Abdennebi M, Guemira F. Deletion polymorphism of glutathione S-transferases M1 and T1 in the Tunisian population. Tunis Med. 2010;88:700-2.

14. Martinez C, Martin F, Fernandez JM, Garcia-Martin E, Sastre J, Diaz-Rubio M, et al. Glutathione S-transferases mu 1, theta 1 , pi 1, alpha 1 and mu 3 genetic polymorphisms and the risk of colorectal and gastric cancers in humans. Pharmacogenomics. 2006;7:711-8.

15. Yu X, Zheng H, Chan MT, Wu WKK. BANCR: a cancer-related long non-coding RNA. Am J Cancer Res. 2017;7:1779-1787.

16. Hayes JD, Strange RC. Glutathione S-transferase polymorphisms and their biological consequences. Pharmacology. 2000;61:154-66.

17. Abou Ghalia AH, Fouad IM. Glutathione and its metabolizing enzymes in patients with different benign and malignant diseases. Clin Biochem. 2000;33:657-62.

18. Ge B, Song Y, Zhang Y, Liu X, Wen Y, Guo X. Glutathione S-transferase M1 (GSTM1) and T1 (GSTT1) null polymorphisms and the risk of hypertension: a meta-analysis. PLoS One. 2015;10:e0118897.

19. Vogl FD, Taioli E, Maugard C, Zheng W, Pinto LF, Ambrosone C, et al. Glutathione S-transferases M1, T1, and P1 and breast cancer: a pooled analysis. Cancer Epidemiol Biomarkers Prev. 2004;13:1473-9.

20. Liu J, Luo J, Wang Y, Li L, Yang S. Predictive potential role of glutathione S-transferases polymorphisms on prognosis of breast cancer. Int J Clin Exp Pathol. 2014;7:8935-40.

21. Piacentini S, Polimanti R, Porreca F, Martinez-Labarga C, De Stefano GF, Fuciarelli M. GSTT1 and GSTM1 gene polymorphisms in European and African populations. Mol Biol Rep. 2011;38:1225-30.

22. Sata F, Yamada H, Kondo T, Gong Y, Tozaki S, Kobashi G, et al. Glutathione S-transferase M1 and T1 polymorphisms and the risk of recurrent pregnancy loss. Mol Hum Reprod. 2003;9:1659.

23. Nair RR, Khanna A, Singh K. Association of GSTT1 and GSTM1 polymorphisms with early pregnancy loss in an Indian population and a meta-analysis. Reprod Biomed Online. 2013;26:313-22.

24. Miller SA, Dykes DD, Polesky HF. A simple salting out procedure for extracting DNA from human nucleated cells. Nucleic Acids Res. 1988;16:1215.

25. Chen CL, Liu Q, Pui CH, Rivera GK, Sandlund JT, Ribeiro R, et al. Higher frequency of glutathione S-transferase deletions in black children with acute lymphoblastic leukemia. Blood. 1997;89:1701-7.

26. Ada AO, Suzen SH, Iscan M. Polymorphisms of cytochrome P450 1A1, glutathione S-transferases M1 and T1 in a Turkish population. Toxicol Lett. 2004;151:311-5.

27. Bansal VK, Rajan K, Sharma A, Paliwal P, Chaubal G, Jindal V, et al. Prospective Case-Control Study to Evaluate the Role of Glutathione S Transferases (GSTT1 and GSTM1) Gene Deletion in Breast Carcinoma and Its Prognostic Significance. Indian J Surg. 2015;77:1067-72.

28. Jaramillo-Rangel G, Ortega-Martinez M, Cerda-Flores RM, Barrera-Saldana HA. Polymorphisms in GSTM1, GSTT1, GSTP1, and GSTM3 genes and breast cancer risk in northeastern Mexico. Genet Mol Res. 2015;14:6465-71.

29. Syamala VS, Sreeja L, Syamala V, Raveendran PB, Balakrishnan $R$, Kuttan R, et al. Influence of germline polymorphisms of 
GSTT1, GSTM1, and GSTP1 in familial versus sporadic breast cancer susceptibility and survival. Fam Cancer. 2008;7:213-20.

30. Song Z, Shao C, Feng C, Lu Y, Gao Y, Dong C. Association of glutathione S-transferase T1, M1, and P1 polymorphisms in the breast cancer risk: a meta-analysis. Ther Clin Risk Manag. 2016;12:763-9.

31. Ramalhinho AC, Fonseca-Moutinho JA, Breitenfeld L.

Glutathione S-transferase M1, T1, and P1 genotypes and breast cancer risk: a study in a Portuguese population. Mol Cell Biochem. 2011;355:265-71.

32. Saxena A, Dhillon VS, Raish M, Asim M, Rehman S, Shukla NK, et al. Detection and relevance of germline genetic polymorphisms in glutathione S-transferases (GSTs) in breast cancer patients from northern Indian population. Breast Cancer Res Treat. 2009;115:537-43.

33. Unlu A, Ates NA, Tamer L, Ates C. Relation of glutathione S-transferase T1, M1 and P1 genotypes and breast cancer risk. Cell Biochem Funct. 2008;26:643-7.

34. Egan KM, Cai Q, Shu XO, Jin F, Zhu TL, Dai Q, et al. Genetic polymorphisms in GSTM1, GSTP1, and GSTT1 and the risk for breast cancer: results from the Shanghai Breast Cancer Study and meta-analysis. Cancer Epidemiol Biomarkers Prev. 2004;13:197-204.

35. Bansal VK, Rajan K, Sharma A, Paliwal P, Chaubal G, Jindal V, et al. Prospective Case-Control Study to Evaluate the Role of Glutathione S Transferases (GSTT1 and GSTM1) Gene Deletion in Breast Carcinoma and Its Prognostic Significance. Indian Journal of Surgery. 2015;77:1067-1072.

36. Helzlsouer KJ, Huang H-Y, Hoffman S, Alberg AJ, Comstock GW, Strickland PT, et al. Association Between Glutathione S-Transferase M1, P1, and T1 Genetic Polymorphisms and Development of Breast Cancer. JNCI: Journal of the National Cancer Institute. 1998;90:512-518. 